\title{
State of Play in the EU-CELAC Common Research Area
}

\section{Estado de la cuestión del área de investigación común UE-ECLAC}

\author{
Martina De Sole ${ }^{1}$
}

Para citar este artículo: De Sole, M. (2019). State of Play in the EU-CELAC Common Research Area. Revista ObIES, 3, 38-43.

Recibido: 5-septiembre-2018 / Aceptado: 8-noviembre-2019

[T]he creation of a Common Research Area does not have to be limited to cooperation on thematic priorities alone. It could also include cooperation on funding researcher mobility, access to research infrastructure and data, and of course sharing knowhow and best practices. Researchers in Europe and in Latin America are already working together every day on the most fundamental questions about life, nature and the advancement of humanity. We have the will, we can build on our past experience, and we can count on the stamina of our researchers to realise our shared vision for the future. We have all we need to build a Common Research Area.

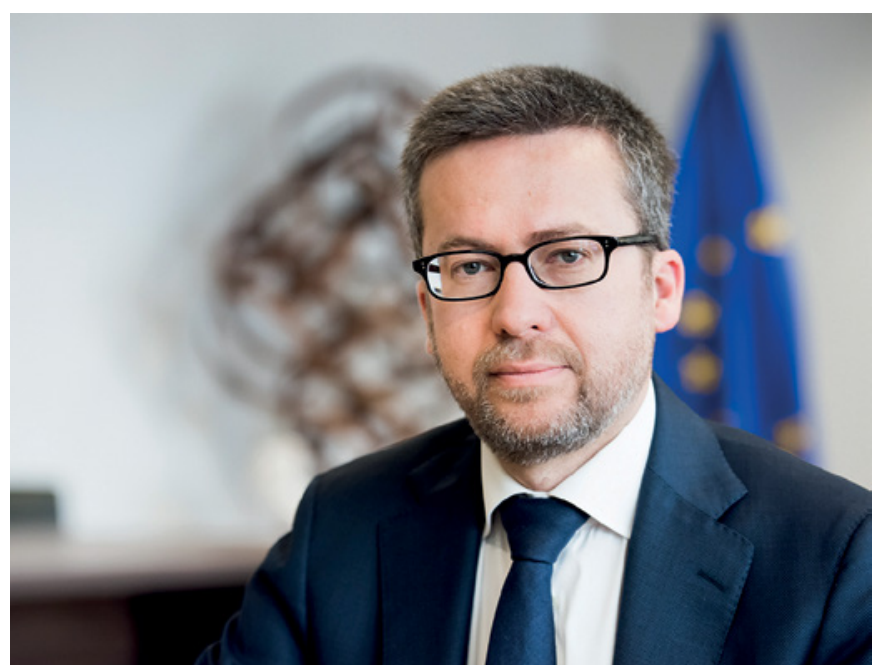

Carlos Moedas, Commissioner for Research, Science and Innovation, 8 June 2015, EU-CELAC Summit, Brussels.

The seed for the ambitious idea of the creation of a Common Research Area between the Europe Union and the countries of the Community of Latin American and Caribbean States (CELAC) countries was planted during the 2010 EU-CELAC Madrid Summit in 2010, when the EU and CELAC launched their Joint Initiative for Research and Innovation (JIRI) and agreed to create an independent chapter for science, research, innovation and technology in a broader EU-CELAC Action Plan, established to consistently foster cooperation at all levels. Then, in June 2015, a call to move towards a Common Research Area was made.

The EU and CELAC have enjoyed privileged relations since their first bi-regional summit, held in Rio de Janeiro, Brazil, in 1999, which established a strategic partnership. Natural partners, linked by strong historical, cultural and economic ties, they co-operate closely at the international level and maintain a close political dialogue at all levels,

1 APRE (Italian Agency for the Promotion of European Research). Email: desole@apre.it 
namely, the regional (the first summit between the EU and CELAC was held in Santiago, Chile, in January 2013), the sub-regional (Central America, the Caribbean, the Andean Community and Mercosur), and, increasingly, the bilateral levels.

The Bi-Regional Policy Dialogue Process in Science and Technology ended its first decade in 2009. During that time, it has advanced its goal of strategic partnership through policy dialogue in regular summits of head of states from the EU and CELAC. In 2010, CELAC was established as a regional bloc of 33 sovereign countries, representing roughly 600 million people. It is now the EU's counterpart for the bi-regional partnership process.

It was only in January 2013 that the new summit process, known as the EU-CELAC was launched.

The cooperation on research and innovation is one of the three pillars of the EU-CELAC bilateral dialogue, together with trade and investment and cooperation, relating to investment and entrepreneurship.

To govern this, the JIRI was established in 2010 with the intention of facilitating bi-regional dialogue on common priorities, as well as encouraging mutual policy learning and ensuring cooperation through biannual action plans. The senior officials meetings (SOMs) with EU-CELAC representatives was established as a part of regular bi-regional dialogue on research and innovation to consolidate EU-CELAC cooperation to implement the JIRI by updating common priorities, encouraging mutual policy learning and ensuring proper implementation and effective cooperation instruments through biannual action plans.

The thematic areas chosen for cooperation at the very beginning were bio-economy, including food security, renewable energies, biodiversity and climate change, ICT and health.

Five thematic working groups were set up under the JIRI to discuss and develop suggestion for joint action. A sixth working group on instruments was also created, and its mandate was expanded at the last SOM in March 2017 to include researchers' careers.
The working groups are co-chaired by representatives of one CELAC and one EU country to ensure that the interests of both groups are taken into account.

The currently existing working groups focus on the following subject areas:

1. Bio-economy, including food security, co-chaired by Argentina and France

2. Renewable energy, co-chaired by Mexico and Spain

3. Biodiversity and climate change, co-chaired by Colombia and France

4. ICT for meeting societal challenges, co-chaired by Chile and France

5. Health, co-chaired by Spain and Brazil

6. Cross-cutting working group on instruments and funding, co-chaired by Portugal and Mexico

The achievements attained and dialogue conducted under the JIRI have led to support for the development of an EU-CELAC Common Research Area to focus on three strategic pillars: researcher mobility, access to research infrastructure and jointly addressing common global challenges, such as climate change and the Zika outbreak.

\section{Common Research Area}

During a JIRI meeting held in March 2017 in Brussels, the Policy Advice Initiative to support CELAC countries in addressing the Sustainable Development Goals through research and innovation was presented as a first instrument to implement activities under the three pillars presented by the European Commission presented.

This new instrument was welcomed, generating clear interest from several countries.

Among additional concrete achievements for each of the pillars of the common research area are the following:

1. Researcher mobility: aside from supporting a more structured bi-regional academic and 
scientific dialogue, the participants committed to developing a regional mobility portal for researchers in CELAC, founded on the previous experience of the EU in the Euraxess portal.

2. Research infrastructure: the SOM endorsed the launch of the working group for policy coordination on research infrastructure (RI) as a first step towards establishing more structured bi-regional cooperation on RI. This group will ensure policy coordination and sharing of best practices in policy development and in mapping of RI.

3. Societal challenges: discussions focused on progress made in the thematic groups and on the large-scale topics potentially related to Horizon 2020, specifically devoted to international cooperation with the CELAC region in the areas of health, sustainable urbanisation and clean urban transport.

\section{Mobility of Researchers}

The EU-CELAC JIRI Seventh Senior Officials Meeting on Science and Technology of 2 October 2017, held in San Salvador, El Salvador, again prioritised researcher mobility. The joint communiqué issued after this meeting states the following:

The Participants welcomed the progress made within the EU-CELAC Common Research Area and agreed on concrete actions under each of its three pillars. 8. Under the pillar of mobility of researchers: a) The Participants stressed the importance of efficient tools and instruments to inform on existing national, regional and European programmes supporting mobility of researchers, such as the European Research Council grants, Horizon 2020 Marie Skłodowska Curie Actions for international mobility and Erasmus+ Programme in Latin America and in the Caribbean. b) The Participants welcomed the opening of the EURAXESS Worldwide Network to CELAC as a way to provide information on mobility, jobs and funding opportunities for CELAC researchers in Europe, and re-iterated their commitment towards the development of a regional portal for researchers in CELAC, based on synergies such as the 'Ibero-American Knowledge Area'.

To assess the state of play for researcher mobility in the EU and CELAC, we interviewed Boryana Yotova, a policy officer for the European Commission Directorate-General for Education, Youth, Sport and Culture, Directorate C, Innovation, International Cooperation and Sport, Unit C.2, Marie Skłodowska-Curie Actions

\section{How many researchers from CELAC countries are participating in MSCA, and how many Europeans are choosing CELAC countries for their research?}

Since 2014, a total of 1005 researchers from CELAC countries have been supported by the MSCA. Argentina has sent the most of its nationals of all 33 CELAC countries, with 292 supported fellows, followed by Colombia (160), Brazil (155), Chile (126) and Mexico (100). In all, 599 research and innovation staff members from CELAC countries have taken part in short-term exchanges in the RISE action, with their main destinations in Europe being Spain, the UK, Italy, Germany and France. In addition, 152 postdoctoral researchers from CELAC countries have obtained MSCA fellowships to work mainly in the UK, Spain and France, while another 254 nationals have gone to other European countries (primarily Germany, Spain and France) for doctoral-level training funded by the MSCA.

Over the same period, CELAC organisations have hosted 525 MSCA fellows, including 13 European² postdoctoral researchers (from Italy, the UK, Spain, Portugal, the Netherlands, Switzerland and Cyprus) under IF, and there are a total of 443 research and innovation staff members seconded by European countries $^{3}$ under RISE (the majority of them are

2 'European' in the meaning of EU Member States and H2020 Associated Countries.

3 Idem. 
Spanish, Italian, French and German). Brazil has hosted the most MSCA researchers and innovation staff members of all 33 CELAC countries (162), followed closely by Argentina (161) and Chile (121).

Furthermore, since 2014, 141 organisations from CELAC countries have participated in 138 MSCA-funded projects, hosting research and training activities on 280 occasions. The distribution of MSCA participation per action is as follows: 245 participations relate to short-term RISE exchanges, 13 to IF Global Fellowships, 12 to doctoral-level ITN and 10 to doctoral and fellowship programmes under COFUND. Out of the total 280 participations of CELAC organisations in MSCA, Argentina ranks first, with 77 participations, followed by Brazil (66 participations) and Chile (51 participations). CONICET (Argentina) is the best-performing CELAC organisation (with 14 participations), followed by the University of Buenos Aires (13 participations) and the University of Chile (12 participations). The vast majority of the CELAC organisations participating in MSCA are in the academic sector.

Argentina, Brazil and Chile are in the top 10 best-performing third countries in MSCA. Out of the total 1887 individual instances of participation as third countries to date, Argentina ranks fifth (with 77 instances), Brazil ranks sixth (66 instances) and Chile ranks eighth (with 51 instances).

\section{How has the establishment of a path towards a Common Research Area influenced the participation of CELAC countries in MSCA and its participation in CELAC countries?}

The Common Research Area is a stepping stone for the overall EU-CELAC partnership. The progress made in the EU-CELAC Common Research Area is being duly monitored at a higher political level, with concrete action being adopted for each of its three pillars.

In relation to the researcher mobility pillar, the MSCA has widely benefitted from this policy framework, which supports the cooperation between the two regions in research and innovation. Overall CELAC participation and engagement in MSCA is constantly improving. The European Commission is strongly committed to exploring new possibilities for cooperation and is ready to assist the responsible authorities from CELAC countries in their efforts to further promote and increase the participation in the programme.

\section{At what stage of development is the EURAXESS CELAC?}

EURAXESS is a European Commission initiative focused on researcher mobility and career development. In 2017, EURAXESS Brazil became EURAXESS Latin America and the Caribbean (LAC).

A team of two dedicated people are based in Brazil, and they support individual researchers in LAC, helping them connect or remain connected with the European Research Area.

The EURAXESS LAC website (http://lac.euraxess. org), publications and mailing lists provide information on jobs and funding opportunities, as well as recent developments of interest to the research community and local events. The team also supports individual researchers with their international mobility projects and institutions from the $\mathrm{LAC}$ region to make the most of EU funding for research and innovation.

Beginning in 2018, the EURAXESS LAC team can now perform missions and organise promotional events on EURAXESS and EU funding for mobility (MSCA and ERC), in Brazil, Argentina, Chile, Colombia and Mexico. Virtual presentations (webinar or videoconference) can be done from any other LAC country.

\section{What is the role of MSCA NCPs in CELAC Countries?}

The following 18 CELAC countries have dedicated NCPs for MSCA at present: Argentina, Bolivia, Brazil, Chile, Colombia, Costa Rica, Cuba, Ecuador, El 
Salvador, Guatemala, Jamaica, Honduras, Mexico, Nicaragua, Panama, Paraguay, Peru and Uruguay. Their contact details can be found on the Horizon 2020 Participant Portal.

The central role of MSCA NCPs is to provide, in the appropriate language, general information on MSCA funding opportunities, to raise awareness of the programme, to guide potential applicants in choosing the most appropriate type of action, to advise on administrative procedures and contractual issues (including such means as info days, conferences, seminars and newsletters), to provide training and assistance on proposal writing and to assist in partner search. The exact portfolio of activities of the MSCA NCPs in the CELAC region varies from one country to another and depends on the given level of experience.

In the framework of the EU-funded Net4Mobility and Net4Mobility+ projects, training and twinning activities for MSCA NCPs have been organised to enhancing their competences and the quality of service they provide. In 2017, a CELAC-dedicated twinning event took place in Bogota, Colombia, gathering experienced and recently appointed MSCA NCPs from Colombia, El Salvador, Honduras, Mexico, Panama, Spain and Uruguay with the main objective of exchanging knowledge and best practices.

\section{What are the next steps?}

Currently, the MSCA are the most international part of Horizon 2020, accounting for more than $50 \%$ of all participation of third countries.

The European Commission is fully committed to building upon MSCA goals and supporting around 15000 non-EU researchers by 2020. It intends to further strengthen the international mobility and cooperation components of the programme under the next Framework Programme.

The next SOM meeting will be held in Brussels before the end of 2019. During the meeting, the progress towards CELAC integration will be monitored, and new and more ambitious cooperation instruments may be proposed if their evaluations produce positive results.

The new President of the European Commission, Ursula von der Leyen, appointed the Bulgarian Marya Gabriel as Commissioner for Innovation and Youth, Research, Education and Culture.

In her In her Mission Letter to Ms. Gabriel, Ms. von der Leyen laid emphasis on the importance of young people, innovators and researchers as 'the best investment in our future', stating that 'Education, research and innovation will be key to our competitiveness and our ability to lead in the transition to a climate-neutral economy and new digital age ...By cooperating across languages, borders and disciplines, we can collectively address the societal challenges and skills shortages that currently exist.' She also referred to the need to 'maximise the potential of our exchange programmes to foster international cooperation in education, research and innovation.

The new commission will enter into force on 1 December 2019, and we expect continuity and advancement in the establishment of an EU-CELAC common research area.

\section{References}

EU-CELAC. (2016). EU-CELAC cooperation in Research Infrastructures.

EU-CELAC. (2017a). EU-CELAC Joint Initiative on Research and Innovation (JIRI). Retrieved from http://alcuenet.eu/assets/Joint\%20Communique_ clean.pdf

EU-CELAC. (2017b). EU-CELAC Joint Initiative on Research and Innovation (JIRI). Retrieved from https://ec.europa.eu/research/iscp/pdf/eu-celac_ joint_032017.pdf

EU-CELAC. (2017c). Roadmap for EU - CELAC $S \& T$ cooperation. Retrieved from https://ec.europa. eu/research/iscp/pdf/policy/celac_roadmap_2017.pdf 
EU-CELAC. (2019). The EU-CELAC Platform for Funding Agencies. Retrieved from https://www. eucelac-platform.eu/
European Commission. (2017). Erasmus+ and MSCA building bridges with Latin America and the Caribbean. Retrieved from https://ec.europa.eu/ programmes/erasmus-plus/news/20171106-erasmus-and-msca-in-latin-america-and-caribbean_en 